\title{
LA BIOLOGIA ARISTOTELICA NELLA PROSPETTIVA DELLE OPERE DE PARTIBUS ANIMALIUM E HISTORIA ANIMALIUM
}

\section{ARISTOTLE'S BIOLOGY IN THE PERSPECTIVE OF THE WORKS DE PARTIBUS ANIMALIUM E HISTORIA ANIMALIUM}

\author{
MiCHA£ OLEKSOWICZ \\ Università Niccolò Copernico, Toruń
}

Recibido: $13 / 11 / 2017 \quad$ Aceptado: $21 / 03 / 2018$

SOMMARIO / RESUMEN

Il magistero aristotelico nella zoologia comparata rimane saldo fino a tempi di scienza moderna, perché non muta in sostanza nei secoli il metodo aristotelico costituito dalla combinazione dei dati anatomici con quelli fisiologici. Pertanto, la straordinaria stabilità assunta dalla biologia aristotelica richiede una riflessione sulle fonti storiche di essa e sui fondamenti del metodo scientifico impiegato da Aristotele nel quadro del suo pensiero biologico presentato in maniera complessiva nelle opere De partibus animalium e Historia Animalium. La riflessione su alcuni aspetti del suo pensiero biologico, ovvero sulla questione della causalità, della sostanza, del metodo dicotomico e comparativo, possa spiegare anche come esse abbiano a un certo punto svolto un ruolo di stimolo e di verifica anche per i temi più generali del suo pensiero filosofico. In effetti, con Aristotele il sapere teoretico della biologia ha conquistato per la prima volta in modo sistematico il grande terreno della natura vivente. Un grande merito di Aristotele filosofo e biologo è quello di aver fondato la scienza della biologia e la filosofia della biologia.

Parole chiave / Palabras clave: Aristotele, biologia, filosofia della biologia, metodo scientifico, storia della scienza. 


\section{ABSTRACT}

The Aristotelian explanations of comparative zoology remained valid until the time of modern science, because during the centuries it had not been changed. The Aristotelian method was characterized by the combination of anatomical and physiological data. The extraordinary stability of Aristotelian biology requires an inquiry into its historical sources and on the foundations of the scientific method employed by Aristotle, in the framework of his biological thought, presented in a comprehensive manner in the works De partibus animalium and Historia Animalium. The inquiry into some aspects of his biological thought, that is on the question of causality, substance, and the dichotomous and comparative method, can also explain how they had, at some point played an inspiring and verifying role in the context of more general topics of his philosophical thought. In fact, with Aristotle's work, the theoretical knowledge of biology had for the first time in a systematic way achieved a coherent structure. A great merit of Aristotle the philosopher and biologist is to have founded the science of biology and the philosophy of biology.

Keywords: Aristotle, biology, history of science, philosophy of biology, scientific method.

\section{INTRODUZIONE}

Verso la metà del IV secolo avanti Cristo le discipline biologiche, dopo la matematica, erano le più avanzate per ricchezza di nozioni. D'altro canto, queste discipline presentavano una certa debolezza scientifica, perché le conoscenze di anatomia e di fisiologia si erano sviluppate soprattutto per opera delle scuole di medicina, e le conoscenze di ordine zoologico erano appannaggio quasi esclusivo di allevatori e pescatori. Due conseguenze emergono immediatamente da questo stato di cose. La prima: un vasto sapere zoologico era in buona parte a disposizione, ma il problema stava nel trovare la metodologia per ordinarlo e interpretarlo mediante un'adeguata teoria. La seconda: i concetti analogico-funzionali di Aristotele fornivano uno strumento perfetto per fare questo tipo di lavoro (Vegetti 1971a, 13-15). Queste circostanze favorevoli spiegano lo straordinario sviluppo assunto dalla biologia nel quadro del pensiero di Aristotele, e spiegano anche come esse abbiano a un certo punto svolto un ruolo di stimolo e di verifica anche per i temi più generali del suo pensiero. La datazione del $P A$ e dell' $H A$, secondo gli studi recenti, non è certa. A questo proposito appare importante considerare la tesi di D. Balme. Egli suggerisce che si dovrebbe evitare un ambizioso tentativo di fare la cronologia dei testi biologici di Aristotele, perché fra le opere biologiche 
e Corpus Aristotelicum ${ }^{1}$ intercorrono assai significative e reciproche interazioni a livello filosofico e scientifico e la loro datazione rimane molto incerta come conseguenza della loro passata rielaborazione (Balme 1987a, 9-20; Vegetti 1971, 487-488).

\section{LE FONTI STORICHE DELLA BIOLOGIA ARISTOTELICA}

Per spiegare la peculiarità della biologia aristotelica si deve domandare quali sono più precisamente le premesse storiche del pensiero biologico di Aristotele. Al primo posto si deve notare la tradizione naturalistica presocratica, dalla quale lo Stagirita deriva la concezione della natura (physis) come realtà dotata di autonoma esistenza e d'intrinseca capacità a finalizzare i processi naturali, realizzando un ordine. Si potrebbero richiamare almeno quattro tratti accomunati nei trattati Peri physeos. Il primo tratto emerge quando si considera che in queste opere viene presentato un sapere circoscritto a ciò che non dipendeva dai tempi $\mathrm{e}$ luoghi, dunque ad aspetti diversi del mondo fisico, a fenomeni ricorrenti nel breve e nel lungo periodo, ai misteri del cosmo. Un secondo tratto indica che la traduzione naturalistica presocratica si caratterizzava da un alto tasso d'intelligibilità, di argomentazione, di condivisione di spiegazioni, scoperte e teorie. La terza peculiarità riguarda il ricorrere di molte tematiche tra un testo all'altro, e l'esplicita competizione fra le diverse teorie. Come quarto costante si può notare la peculiare "inutilità" del sapere, cioè di un sapere che non ha immediatamente applicazioni pratiche e quotidiane (Rossetti 2004, 95-110).

In effetti Aristotele ha sviluppato tutto il patrimonio della physiologia muovendosi sulle tracie delineate dalla grande tradizione naturalistica. Da un lato lo Stagirita si avvicinava al suo versante siciliano, fondato da Empedocle e continuato da Filistione (la dottrina della composizione della materia dai quattro elementi e delle quattro proprietà attive) e anche da questa fonte prendeva la nozione della centralità del calore nella vita organica. Proprio questo privilegiare dal punto di vista fisiologico le "sedi" corporee del calore stesso, cioè il cuore e il sangue, dipendeva sia dalla tradizione empedoclea come dalla tradizione pitagorica, rappresentata da Filolao, e comportava l'assegnazione al "principio" del calore un ruolo centrale nella vita psichica. Dall'altro lato, dalla physiologia di origine ionica (Diogene di Apollonia), egli accettava l'importante ruolo dell'aria, del respiro (pneûma) nei processi vitali. Anche nell'intento logico-classificatorio

1 Abbreviazioni: $P A$ - De partibus animalium; $H A$ - Historia animalium; $G A$ - De generatione animalium; Met. - Metafisica; Phys. - Fisica; APr. - Analitici Primi; APo. - Analitici Posteriori; 
e metafisico di Aristotele si può vedere l'influsso del tardo pensiero platonico che promuoveva un ordine di ricerche in campo zoologico e botanico. Inoltre, si trova nel pensiero aristotelico un progressivo allontanamento dalla linea encefalocentrica di Alcmeone-Anassagora-Ippocrate e dalla linea platonica che riconosceva una doppia centralità, del cervello nelle funzioni psichiche e del cuore nelle funzioni organiche (Berti 2004, 174; Vegetti 1971a, 15-18).

$\mathrm{Al}$ secondo posto si deve notare la tradizione delle grandi scuole di medicina alle quali Aristotele poteva riferirsi. Lo Stagirita si rifaceva a opere ippocratiche (Articolazioni, Fratture, Ferite nella testa, Natura dell'uomo) per le informazioni sul sistema osseo e sul sistema vascolare (Vegetti 1971a, 18-19). Dall'opera eclettica De Victu lo Stagirita poteva derivare alcune rare informazioni zoologiche e accenni d'indagine psicosomatica sui rapporti fra corpo e funzioni psichiche. La discussione sugli umori, e in particolare sulla bile, derivava dal trattato sui Luoghi nell'uomo. Il sapere medico era una fonte molto importante ma presentava anche gravi limiti. Nella medicina di quel tempo prevaleva l'orientamento patologico e anche in quella cultura era data la difficoltà di sezionare cadaveri. Inoltre, Aristotele declassava piuttosto la medicina alla tecnica applicativa, terapeutica, e subordinata a livello teoretico della biologia (Vegetti 1971b, 91-92).

La terza fonte di conoscenze naturalistiche, prevalentemente empiriche, viene dall'esperienza, dal mondo delle technai, cioè dalla pratica di allevatori, cacciatori, agricoltori, pescatori. Tuttavia, questa intuizione non sarebbe imposta se non fosse stata già elaborata, proprio nei nuclei importanti per questa problematica, epistemologia dello Stagirita. È ben noto che sia il libro I della Metafisica sia gli Analitici (APo. II.19) mettono l'empiria, l'esperienza, alla base del sapere scientifico. Evidentemente non si tratta lì dell'esperienza nel senso del moderno metodo sperimentale, ma proprio come competenza pratica, che deriva dagli "esperti", cioè non l'esperienza metodicamente organizzata, ma l'esperienza che si forma nel corso della vita, e che ha un significato anzitutto pratico (cf. Etica Nicomachea VI.8, II; HA 573a13-4). Dunque, sulla base di questo assetto teoretico, Aristotele poteva "uscire" dall'ambito delle technai tradizionalmente accettate (la medicina, la matematica, l'architettura, la retorica) per riconoscere la conoscenza d'empiria anche da parte delle pratiche quotidiane degli allevatori di bestiame (HA III.10, 17, 20), degli agricoltori, dei cacciatori, dei pescatori ( $H A$ IV.7, V.16) e dei diversi mestieri (cf. $H A$ 587a9-22; $H A$ V.15). In questo modo egli poteva cogliere questa somma imponente d'informazioni zoologiche, che hanno fornito la base dell' $H A$ e di tutta la posteriore teorizzazione biologica. In effetti, Aristotele ha fatto un assiduo lavoro d'interviste e d'inchieste per raccogliere non solo una grande quantità di dati, ma anche per quei linguaggi semispecializzati, i metodi osservativi ed euristici che creavano la base sia dell' $H A$ sia 
il punto di partenza per la ricerca compiuta poi nel PA (Lloyd 1987, 53-63; Berti 2004, 175).

Non si possono dimenticare anche non di meno i due importanti fatti dalla biografia di Aristotele. Egli era figlio di Nicomaco, che lavorava come medico personale di Aminta II di Macedonia. Rimasto orfano in minore età fu adottato da un parente, Prosseno di Atarneo, ma sicuramente il periodo vissuto con il suo padre naturale gli ha dato le possibilità di conoscere in qualche misura la medicina. Forse già con Nicomaco sezionava i cadaveri e usava il metodo comparativo sugli organismi viventi (anatopatologia). Se fosse stato così, da casa sua Aristotele avrebbe ricevuto una forte convinzione della necessità di avere un dato concreto per spiegare la realtà. Nel caso della dissezione si tratta di un passo scientifico senza precedenti, dove "l'osservazione metodicamente regolata comporta di norma il ricorso a quella dissezione anatomica che era stata ignorata dai predecessori, e che avrebbe invece costituito l'asse portante del sapere zoologico di Aristotele" (Berti 2004, 178).

D'altra parte, dopo la morte di Platone, Aristotele aveva abbandonato Atene e si era recato alla corte di Ermia ad Atarneo dove si trovano i platonici. Ermia trova, per lui e i suoi compagni, la sistemazione ad Asso, dove probabilmente conosce Teofrasto. E proprio con quest'ultimo lo Stagirita è andato a Mitilene, nell'isola di Lesbo. Lì si consolida in quasi dieci anni una forte collaborazione tra i due filosofi, che come frutto ha portato un grandissimo materiale empirico raccolto in $H A$. Da quanto è stato detto sino ad ora, risulta un'immagine di Aristotele sia come attento osservatore e direttore delle ricerche (specialmente negli anni di Asso e Mitilene in cui egli raccolse la gran parte delle informazioni zoologiche e osservò di persona numerose dissezioni: su piccoli animali marini, su qualche quadrupede e anche sul feto umano abortito o sul cadavere di neonati), sia come teorico della scienza (Vegetti 1971a, 20-23).

\section{SPIEGAZIONE CAUSALE E LA QUESTIONE DELLA SOSTANZA}

Nella spiegazione del metodo aristotelico si deve rendere conto che la scienza aristotelica è essenzialmente una conoscenza per cause. Conoscere le cause significa conoscere la cosa stessa, giacché "l'essenza dell'oggetto s'identifica con il perché l'oggetto sia" (APO. 90a15). Nel PA Aristotele pone la domanda se lo "studioso della natura deve osservare prima i fenomeni relativi agli animali, per poi spiegare il perché e le cause, oppure procedere in qualche altro modo" ( $P A$ 639b6-10). La risposta affermativa è data in un passo dove lo Stagirita spiega che "il punto di partenza debba consistere nel raccogliere i fenomeni relativi a ciascun 
genere, e che si debbano poi esporre le loro cause e trattare della generazione" ( $P A$ 640a13-15). Cioè, nell'orizzonte aristotelico i fenomeni non hanno bisogno della giustificazione teoretica, ma analizzandone le cause si mette in luce la loro struttura essenziale.

Le cause insite nei processi naturali (PA 639b11-2) sono rappresentate nel pensiero aristotelico dalla classica identificazione di esse (Met. I.3; Phys. II. 3.7), ma la prima questione di fondo riguarda il problema dell'importanza della causa finale. Infatti, Aristotele dichiara che "il processo di formazione infatti è finalizzato alla cosa, ma la cosa non è finalizzata al processo" ( $P A$ 640a18), oppure che "vi è più finalità e perfezione nelle opere della natura che in quelle della tecnica" (PA 639b18), e che "la natura fa tutto in vista di un fine" (PA 641b12). Evidentemente la causa finale ha un'importanza notevole per Aristotele e rappresenta la direzione e il senso del processo naturale, sia un mutamento interno al vivente (kinessis; PA 641b5-8) sia la riproduzione interna all'ousia o specie (genesis). Per Aristotele lo scopo non è solo in funzione del processo, ossia della causa agente. Secondo lo Stagirita il fine è il logos (l'essenza), cioè la determinazione necessaria perché una cosa sia quello che è. Dunque, il logos si pone come principio dei processi, in quanto è il loro fine ( $P A$ 639b14-16; cf. GA 715a4-6). Per Aristotele il fine è il logos, ciò significa dire che il fine è l'ousia, ovvero che ogni processo che si svolge all'interno di una realtà vivente è tale da far sì che essa sia quello che essa essenzialmente è (Vegetti 1971c, 506). Dopo aver puntualizzato che il fine di ogni ousia, specie, consiste nell'essere compiutamente se stessa, emerge che l'idea di processo e ousia non è percepita da Aristotele in modo statico, ma piuttosto dinamico. Il fine permette alla cosa di essere quello che è, di realizzare la sua essenza. In questo senso l'essenza della cosa è la funzione della cosa stessa, e dunque la funzione determina la forma della cosa (PA 642a9-13; $694 \mathrm{~b} 13 ; 645 \mathrm{~b} 14-20)$. Nei viventi la funzione consiste nel garantire la conservazione della specie. Altre volte, invece, il rapporto tra telos e necessità naturale, sembra essere invertito, sicché la materia appare la premessa dalla quale si conclude al telos (PA 663b20-24). Questo non significa che Aristotele passa dal finalismo al meccanicismo materialistico, ma che fa un'indagine da punti di vista interpretativi diversi, secondo i dati a disposizione (Vegetti 1971c, 515) ${ }^{2}$.

Accanto a questa compenetrazione di ousia e telos sta il ruolo della causa efficiente e materiale. Secondo Aristotele la natura vivente, in quanto ousia e telos, è anche principio di mutamento di se stessa ( $P A$ 641a25-7). La natura (physis) è definita da Aristotele in due sensi: "quello di materia e quello di essenza, ed è

2 Per uno studio della teoria aristotelica delle cause e della sua attualità teorica nel contesto di odierna questione dell'emergenza e di downward causation cf. Tabaczek, 115-134. 
questa seconda, in quanto anche causa motrice e fine" (PA 641a25). Qui la physis è intesa come l'insieme delle cose che hanno in sé il principio del proprio mutamento - la realtà automotrice (cf. Phys. 193a28-30; Met. V.4). Di conseguenza la causa efficiente è riassorbita nel campo della vita animale dalla prevalenza in essa dell'anima, la quale non è che il complesso di funzioni psichiche che caratterizzano la vita animale in quanto tale, e sono connesse alla corporeità. Per questo motivo Aristotele aggiunge che quando si concepisce la natura nel senso dell'essenza occorre intendere "l'anima dell'animale, o nella sua totalità o in una sua parte" e "chi studia la natura dovrà parlare più dell'anima che della materia, tanto più che la materia è natura grazie alla prima, piuttosto che il contrario" ( $P A$ 641a26-27). Dunque la natura si definisce nella tensione fra due lati, di cui l'uno è principio, l'altro strumento o sostrato $(P A$ 642a17). In un altro passo Aristotele, considerando i due tipi di necessità - quella eterna, incondizionata e quella condizionale -, definisce la materia nell'ambito di necessità condizionale, perché essa è condizionata nella sua struttura e nella sua funzione dal sistema ousia-telos (PA 639b21-30; cf. GA 789b2-15). Ora appare chiaro che l'apodeixis propria della scienza della natura è diversa dalle altre scienze teoretiche (la matematica, la teologia). Le ultime hanno come oggetto enti che si definiscono nel loro essere ( ò ôv), invece la prima enti determinabili nel loro processo, il cui principio è ciò

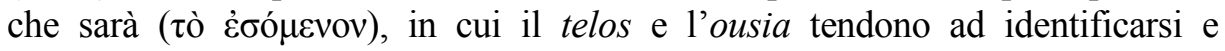
costituiscono la manifestazione dinamica dell'essenza - la dinamicità finalizzata (PA 639b30-640a4; 641b10-12; cf. GA II. 6) (Balme 1987b, 275-285; Balme 1987c, 291-312).

Da quanto è stato detto finora, risulta che, secondo Aristotele, le cause nel campo biologico esprimono la struttura multidimensionale di ogni realtà esistente. Secondo Aristotele ogni ousia (ogni singola specie) esaurisce in sé sia le proprie cause sia i propri processi di formazione e di trasformazione, e pertanto l'ousia è autofondata e autocentrica. Dunque, per Aristotele ogni realtà vivente è finalizzata alla propria autoconservazione, è esclusa ogni interrelazione fra le diverse ousiai (individui o specie del vivente). Se la physis non può essere concepita come rete di connessioni fra ousiai, essa va interpretata per indicare "l'insieme delle cose che sono per natura" (Vegetti 1987c, 508-509). E proprio in questa espressione torna una dimensione unitaria di physis, non già metafisica, né finalistica, ma analogica. Di conseguenza la physis, che si divide in diverse regioni dei viventi, le diverse specie degli animali che, non comunicano essenzialmente fra loro, comunque presentano le analogie delle strutture. Questa possibilità di studio analogico di una regione della realtà, da un lato fonda l'uso del metodo espositivo che dichiara la mappa della regione e dall'altro lato mette in luce "principi propri" della regione esaminata, che esprimono le analogie strutturali in base alle quali si 
distinguono le diverse ousiai. Descrivere le analogie strutturali è proprio il problema della zoologia comparata dei libri II-IV del $P A$.

\section{METODO SCIENTIFICO}

Il metodo aristotelico usato nelle prime ricerche biologiche, secondo M. Vegetti, può essere identificato come un metodo euristico dell'inferenza semeiotica. Come si è già detto questo metodo è divenuto il principale strumento euristico delle grandi technai del V. sec., ed era accompagnato dalla riflessione filosofica che passava da Alcmeone fino al primo Platone. Questo metodo consisteva nello scoprire il valore d'indizio (tekmērion), di sintomo, cioè la funzione semeiotica dei singoli dati di esperienza (sèmeion) e poi concludere con le inferenze (tekmairesthai) sia sui dati ancora ignoti, sia sulla totalità del sistema, sia sulle previsioni degli sviluppi dinamici del sistema. Dunque, questo metodo mostra esplicitamente come dall'attività pratica e dall'accumulazione delle conoscenze empiriche, attraverso il metodo euristico e d'inferenza empirica, si passi all'elaborazione dei dati ${ }^{3}$. In effetti sembra che la struttura metodica dell' $H A$ sia fornita dall'uso del metodo semeiotico delle technai. Inoltre, è bene rilevare la presenza nell' $H A$ di alcune considerazioni di tipo statistico, espresse in una celebre formula "per lo più", e basate sulla frequenza dei fenomeni e delle loro connessioni. Si dovrebbe notare che nel maturo pensiero aristotelico, usando i termini moderni, la frequenza dei fenomeni è garantita piuttosto a priori che a posteriori. Questo avviene, perché la regolarità di physis è basata sulla costanza di strutture delle ousiai che fondano la natura. Ma nell' $H A$ si potrebbe notare un quadro del pensiero non ancora condizionato dalla nozione dell'ousia. Ci sono i passi (HA 538a30-538b2; 571a26-7) dove il verbo stochazesthai sta a qualificare il "per lo più", e lo qualifica come un risultato dell'elaborazione di tipo statistico, che viene dall'esperienza dei pescatori, e non come il riflesso fenomenico della stabilità della natura. Alla luce di questo passo appare chiaro che l'empirismo aristotelico, già dalla prima ricerca biologica condotta nell' $H A$, consiste nello sforzo di stare in contatto con il phainomenon, e nella critica, nel controllo dei dati di osservazione. Invece sembra che nel $P A$ l'accento sarà messo piuttosto nella ricerca

3 Va notato che esiste discordanza fra il metodo semeiotico dell' $H A$ e del $P A$; p.es., la questione della longevità dei cervi nell' $H A(578 \mathrm{~b} 23-26)$ viene attestata da una lunga tradizione dei racconti, delle opinioni, mentre nel $P A$ l'opinione degli antichi è accettata nel contesto di un rapporto causale fra l'assenza di bile e la longevità dei cervi (cf. $P A$ 677a30-32). Pertanto sembra che la debolezza del metodo semeiotico consisterebbe nell'uso di sēmeia come il tipo d'indicazioni di qualche processo (PA 667b1; 669b4; 672b28; 679a21; 680a31; 681a7), ma senza mostrare le evidenze dirette per esse (Lennox 2001b, 261). 
ontologica ed epistemologica della giustificazione del dato d'osservazione (Vegetti 1971b, 92-95, 97-101).

Va notato che il ruolo specifico della spiegazione causale per lo Stagirita non sta nella costruzione di una spiegazione teoretica in quanto tale, ma sta nel passaggio dal livello dell'osservazione dei dati e delle regolarità empiriche a quello della spiegazione causale, cioè della teoria scientifica (PA 646a8-11). In tal modo Aristotele fa un passaggio dal livello del "che" (hoti) al livello del "perché" (dioti) proprio grazie alla spiegazione causale (cf. PA 652a19-21). Tuttavia, si può costatare che la teoria delle cause è semplificata dallo schema classico. Si tratta piuttosto di due punti di vista - del "necessario" (anankaion) e del "meglio" (beltion, beltiston). In effetti la causa efficiente si congiunge con la materiale e il fine si congiunge con la forma. Questo accade perché la corporeità degli animali è collegata con il campo dei processi necessitati dalla consequenzialità non finalizzata della materia. Contemporaneamente l'adeguamento dei processi allo svolgimento delle funzioni si esprime attraverso l'unificazione della causa formale e finale (Basti 2008, 29-51).

Sembra che la teleologia non sia per Aristotele una modalità privilegiata per la spiegazione dei fenomeni naturali ma proprio un strumento d'indagine molto potente nel campo biologico che rende possibile mettere in luce il rapporto dell'organismo con le sue funzioni. In un passo che riguarda la spiegazione aristotelica delle corna ( $P A$ 663b20-24) si vede la doppia prospettiva della comprensione scientifica: l'importanza della dimensione statico-strutturale ("natura necessaria") e della dimensione dinamico-funzionale ("natura secondo l'essenza") dell'unica realtà - l'ousia. Questa comprensione non può essere concepita solo in modo teoretico ma deve venir condotta mettendo in opera punti di vista diversi che, come si è visto, consentano di analizzare la struttura dell'oggetto (Vegetti 1971c, 518, 526-528). Dunque, Aristotele ha creato due cruciali categorie per la scienza biologica: la categoria della necessità e della finalizzazione, ma la prevalenza dell'una sull'altra dipende esclusivamente dalla loro capacità di interpretare $\mathrm{i}$ dati d'osservazione. La categoria della finalizzazione ben serve a spiegare il principio biologico dell'adattamento, secondo il quale l'organo si adatta alla funzione che gli compete (PA 694b13-4). L'altra categoria, della consequenzialità inerziale dei processi corporei, spiega che la realizzazione del fine non è mai incondizionata ma ha sempre un adattamento dell'organo al fine, e questo adattamento è condizionato dai dati strutturali della materia vivente, la quale costituisce la base della funzione. Questo significa l'interdipendenza fra materia e fine, tra la struttura e la funzione ( $P A$ 658a23-4; 687a16-7). Per Aristotele queste coppie delle categorie sono indispensabili alla costruzione della teoria scientifica, perché la finalità sia condizionata dalla struttura dell'ousia. Ma 
egli anche dichiara che la finalizzazione può essere talvolta inutile e fuorviante, quando la si cerchi essa nei processi non funzionali, p.es. la natura dei residui ( $P A$ 677a15-18), dove dovrebbe solo rimanere la spiegazione "per necessità" materiale. In un altro passo ( $P A$ 685b11-15), che riguarda lo studio degli ordini di ventose negli ottopodi, Aristotele si distacca dalla spiegazione finalistica (beltiston), e dà invece luogo a una spiegazione secondo il concetto della necessità (anankaion). Evidentemente la biologia di Aristotele non è né finalistica né meccanicistica, ma l'unica cosa che conti per la scienza è quella di comprendere in modo più adeguato l'ousia (Cooper 1987, 243-274).

Accanto alla grande forza esplicativa della spiegazione causale si possono vedere i limiti di quest'applicazione. Essi stanno proprio nella pretesa di Aristotele di ricostruire ogni particolare aspetto dell'ousia rivelato nell'osservazione empirica per mezzo delle strutture causali (cf. PA 680b9-29). Per Aristotele ogni fenomeno sia immediatamente integrabile nella spiegazione teoretica, perché esso è sempre verace. Tuttavia, un'osservazione imperfetta, fallace o parziale, basta a mettere in crisi tutta la teorizzazione, tutto l'edificio esplicativo. La fedeltà all'osservazione costruisce anche il forte limite della sua biologia, ciò trova la sua conferma nel frequente uso del termine eulogon, che vale a dire che i fatti stanno come stanno, in linea a quanto razionalmente ci si deve aspettare. A questo punto appare interessante domandare se la fedeltà all'osservazione empirica, avendo i suoi certi limiti, doveva necessariamente portare Aristotele a escludere l'uso di qualche metodo euristico di quantificazione e previsione dei fenomeni sulla base della matematizzazione dell'esperienza. Infatti, si potrebbe immaginare, che il pensiero aristotelico nel campo biologico poteva evolvere di più sulla traccia delle tendenze statistico-probabilistiche, concepite come collegamento fra l'empirismo dell'ippocratica Antica Medicina (l'impossibilità della rigorosa esattezza, akribeia) e la convinzione platonica del "più e meno" ( $\mu \tilde{\alpha} \lambda \lambda \mathrm{ov} / \tilde{\tilde{\eta}} \tau \tau o v$ era introdotto nel Filebo 24 A-B) (Vegetti 1971b, 116).

\section{ABBANDONO DEL METODO DICOTOMICO?}

Nel $P A$ si svolge una polemica aristotelica con il metodo della dicotomia platonico-accademica, la quale era utilizzata da Aristotele nell' $H A$. La sua critica di questo metodo occupa quasi la metà del libro I del $P A$. La dicotomia, alla metà del IV secolo era considerata come il culmine della dialettica e come lo strumento conoscitivo universale funzionante in tutti campi del sapere, ma particolarmente in quello del sapere naturalistico (Cherniss 1944, 1-82). L'elasticità della dicotomia deriva dal fatto che il procedimento dicotomico dipende dal campo biologico su cui si opera le divisioni, e dalla scelta del punto di vista e delle relative 
differenze. Dunque, il valore euristico della dicotomia sta nel punto di vista comparativo, dove il genere degli animali, scoperto piuttosto sulla base del linguaggio delle technai che dello studio delle differenze delle specie, serve da campo dicotomico di base. Di conseguenza, le strutture dicotomiche sono, per loro natura, aperte e provvisorie, cioè mostrano un'estrema funzionalità e apertura al tipo di ricerca che si sta svolgendo. A questo punto risulta essenziale enfatizzare che la conquista del metodo comparativo è stata già fatta da Aristotele nell' $H A$, perché in essa quel metodo diventa una naturale conseguenza dell'uso dell'approccio dicotomico, cioè focalizzarsi sulla determinazione analitica delle differenze e delle similarità presentati dagli animali, ciò che poi viene messo negli schemi dicotomici ordinativi (HA V.4; 566b8-13; VI.36; VIII.2). Le due grandi categorie di organizzazione degli animali, la differenziazione (la differenza, diaphorà) e l'identificazione (la similarità, homoiotess) agiscono a tre livelli, perché si potrebbero distinguere tre diversi modi di differenziazione e di similarità degli animali: il primo, fra individui all'interno della specie-forma (eidos); il secondo, fra le specie, all'interno del genere (il livello del "più e meno", cioè le differenze quantitative); il terzo, fra generi, all'interno del mondo dei viventi, cioè il livello dell'analogia. La specie e il genere dal punto di vista tassonomico non avevano alcun valore fisso o determinato, e dunque solo in funzione delle similarità e delle differenze, si poteva stabilire una gerarchia fra genere e specie (Lennox 2001a, 39-71; Pellegrin 1987, 313-338; Vegetti 1971b, 115-116).

Probabilmente Speusippo, negli Homoia, cercava di trasformare la dicotomia in ordinamento naturale, cioè passare dalle relazioni diairetiche in contenuti dell'esistenza stessa per fare la rappresentazione gerarchica del mondo naturale (Cherniss 1944, 44-48). A questo punto appare chiaro che la critica aristotelica era indirizzata verso le idee di Platone, ma probabilmente non tanto nel suo versante noetico-ideale di Platone quanto in una sorta di metafisica rappresentata da Speusippo. In altre parole, la dicotomia in quanto strumento logico generale viene rifiutata a vantaggio della nuova teoria sillogistico-dimostrativa (cf. $A P r$. I.31, APo. II.5). Invece, in quanto strumento d'organizzazione e d'interpretazione del mondo naturale, privo di implicazioni ontologiche ma usato come metodo comparativo, viene riconosciuta come strumento valido ed efficace. In tal modo, il punto di vista comparativo ampiamente usato nell' $H A$, elaborato non solo come la rassegna delle informazioni ma come il procedimento kath'hekaston (HA 491a5) che conduce alla trattazione analogico-comparativa, s'incontra con il nuovo quadro teoretico del $P A$, dove Aristotele sviluppa la teoria della ousiaspecie. Benché il metodo dicotomico viene fortemente ripensato da Aristotele nel PA (cf. Categorie, cap. 13; Topici, I.17; VI.6; Met. X; APo. 91b32-5), si potrebbe concludere che il suo indiscusso contributo sta nello sviluppo sistematico del 
metodo comparativo-analogico, come conseguenza di un contatto con ricco materiale empirico e con il metodo dell'inferenza semeiotica delle technai (Vegetti 1971b, 102-104, 119-120).

Lo sfondo per il distacco aristotelico dal metodo dicotomico si basa sugli argomenti espressi nel testo di Met. VII.12-14, dove Aristotele spiega che il genere non ha esistenza reale ma analogica. L'unica realtà spetta all'ousia-specie e tutte le differenze che applica la dicotomia hanno solo una consistenza verbale, sicché solo l'ultima di esse indica l'essenza della cosa, cioè costituisce la sua definizione (Met. 1038a19-20). Le critiche espresse nel $P A$ sviluppano quest'idea, ma con un'importante osservazione. Aristotele nel $P A$ ribadisce che soltanto l'ultima differenza è significativa ( $P A$ 642b7-9), perché la forma essenziale, che definisce la specie, è una e indivisibile ( $P A$ 643a2), e da ciò consegue che ci sono tante differenze reali quante sono le specie indivisibili degli animali ( $P A$ 643a7). Tuttavia, da quanto è stato detto sinora, secondo Aristotele, deriva una conseguenza la quale non era esplicitata nella Metafisica: ognuno dei generi è definito da molte differenze insieme ( $P A$ 643b12-3). Dunque, l'unità del genere è divisa secondo molte differenze e non dicotomicamente ( $P A$ 643b23-4). Le altre argomentazioni aristoteliche possono essere riassunte nel modo seguente: 1 . sarebbe assurdo che le specie di qualsiasi genere debbano essere in numero di potenza di due (PA 643a20-24); 2. il metodo dicotomico comporta lo smembramento dei generi naturali, ciò alla fine determina l'inclusione artificiale di gruppi appartenenti allo stesso genere ma messi in classi diverse, cioè sovrapposizione al carattere euristico e artificiale quello naturale e ontologico del procedimento analogico ( $P A$ 642b10-20); 3. sarebbe difficile seguire il procedimento per privazione del metodo dicotomico, perché "non v'è differenza nella privazione in quanto privazione: giacché è impossibile che vi siano specie di ciò che non è" ( $P A$ $642 \mathrm{~b} 22-3)$.

Al rifiuto della dicotomia Aristotele fa l'esplicazione dei procedimenti della vera scienza. Perché la natura si manifesta per quello che è, occorre "seguire la via indicata dai più" ( $P A$ 643b10), cioè ascoltare le opinioni e il linguaggio naturale degli uomini. Inoltre, occorre studiare ogni specie-ousia, non con una sola differenza, ma con più differenze applicate simultaneamente ( $P A$ 643b28644a11). In effetti, Aristotele abbandona il procedimento teorico che parte dal genere e ribadisce che "il meglio sarebbe poter considerare separatamente le realtà individuali e non divisibili quanto alla specie, come si fa per l'uomo; allo stesso modo si procederebbe, non per gli uccelli, perché questo genere ha specie, ma per qualsiasi specie indivisibile degli uccelli, ad esempio i passeri, le gru e così via" ( $P A$ 644a29-33). Alla luce di questo passo si può comprendere perché non vi sia nel $P A$ nessun tentativo di creare una vera classificazione degli animali, ma 
piuttosto la sua classificazione è approssimativa e mutevole come un abbozzo funzionale. Procedendo secondo il modo di una classificazione rigida, "capiterebbe di ripetersi spesso sul medesimo attributo, a causa della sua appartenenza a più specie secondo un rapporto comune" e dunque per le ragioni espositive Aristotele considera come opportuno e ragionevole esporre gli attributi comuni a ogni genere, cioè le determinazioni delle singole specie ( $P A$ 644a34644b15). In tal modo, come si è detto, la dicotomia è respinta sia come strumento euristico con eventuali implicazioni metafisiche sia come metodo di rigida classificazione (Vegetti 1971c, 522-524).

Dopo aver puntualizzato in che cosa consiste la specificità del metodo dicotomico e un tipo di elaborazione fatta a essa da Aristotele, emerge in modo più diretto la domanda se Aristotele ha abbandonato il metodo dicotomico. Sembra di no (Lennox 2001a, 76-82). È bene rilevare che il concetto del "problema" gioca un ruolo centrale negli Analitici, in modo particolare nelle due discussioni - APr. I.26-31 e APo. II.14-17 - molto correlate con se stesse. In APr. I.27-30 Aristotele descrive il procedimento di selezionare le premesse relative al discusso problema, in modo particolare la scoperta del termine medio. In APo. II.14-17 le proposizioni sono considerate come gli oggetti dell'indagine, per stabilirli come explananda delle dimostrazioni. In altre parole, le proposizioni non hanno solo il ruolo di tagliare il contesto della discussione, ma esprimono la ricerca scientifica, cioè rivestono la forma della domanda "perché". Già queste discussioni negli Analitici - il problema della scelta delle premesse, investigazione "perché" - si differiscono dalle discussioni nei Topici (VIII.2 158a14-18) sui problemi dialettici. Per questo motivo Aristotele negli APr. I.1 24a22-25 fa la distinzione fra le premesse dimostrative e le premesse dialettiche del ragionamento. Nel primo caso si assume un termine della proposizione, nel secondo caso si discute sull'alternativa contraddittoria. Dunque, la risposta alle premesse dialettiche può essere "sì" o "no", ma alle domande di tipo "perché": no. In tal modo J.G. Lennox suggerisce di mettere queste considerazioni di Aristotele in uno schema, dove le domande e le risposte si pongono a due livelli.

Il livello 1: comune al contesto dialettico e scientifico.
La domanda:
ci sono $\mathrm{S}(\mathrm{o} \mathrm{P})$ o no?
Tutti S sono P o no?
La risposta:
ci sono $\mathrm{S}(\mathrm{P})$
Tutti S sono P

Il livello 2: limitato al contesto scientifico.
La domanda:
che cosa è $\mathrm{S}(\mathrm{P})$ ?
Perché tutti S sono P?
La risposta:
$\mathrm{S}(\mathrm{P})$ sono $\Psi$
Tutti S sono P perché $\Phi$ 
Si può interpretare che il metodo dialettico fornisce le risorse per muoversi dalle domande del livello primo alle risposte di quel livello. Al livello primo le differenze fra indagine dialettica e scientifica non sono del tipo logico, ma del tipo epistemico, cioè dipendono dalle diverse evidenze che stanno alla base delle risposte. Di conseguenza, nel contesto scientifico, nonostante che la struttura delle domande e delle risposte sia la stessa come nel contesto dialettico, le risposte sono raggiunte grazie alla spiegazione causale, via il termine medio. Al livello secondo si assumono le risposte vere dal livello primo e poi si procede nella spiegazione causale delle proprietà degli oggetti studiati. Tuttavia, può sembrare che queste distinzioni siano fatte in modo arbitrario, perché come si è detto, il metodo dialettico, dicotomico è stato usato per mostrare le differenze e discutere i problemi delle definizioni dei generi, delle specie (Top. I.9 103b24-27). Dunque, esso non esclude l'indagine sulle cause e sugli attributi degli animali. Ma secondo Lennox questo dubbio non coglie il fulcro della discussione aristotelica, nella quale lo Stagirita vuole spiegare che ciò che differisce questi due tipi d'indagine, non è l'oggetto del problema, ma la forma del problema. Dunque, queste due indagini sono state in modo consapevole distinte, ma non per escludersi reciprocamente, ma per sottolineare l'intreccio che esiste fra di loro. In APo. II.2 89b2529 Aristotele spiega che quando domandiamo "è il caso che $\mathrm{S}$ è P?", in realtà chiediamo "perché è il caso che?". Anzi, chiediamo se c'è o no un termine medio per S-P (b37-38), e anche quando cerchiamo la ragione perché "S è P", facciamo la precisa domanda su quale termine medio esiste fra loro.

In conclusione, per Aristotele anche le domande dialettiche implicano le domande sul fondamento causale. Evidentemente il metodo aristotelico si concentra sul cogliere i problemi grazie alla divisione, dicotomia fatta in modo metodico. Se la divisione è fatta così, si mostra che lo stesso logos spiega che la studiata proprietà appartiene alle diverse specie, perché esse hanno la stessa forma (logos), la forma che appartiene in modo essenziale al loro genere comune. Questo procedimento metodico permette di identificare i problemi più fondamentali e a intrecciare l'esposizione dialettica con la spiegazione causale. Pertanto, sembra che Aristotele non abbandona il metodo dicotomico della dialettica platonica, ma lo usa come una parte della sua indagine scientifica nel campo biologico.

\section{I PRINCIPI DEL METODO COMPARATIVO}

Nel $P A$ il riconoscimento della specie-essenza in quanto nucleo ontologico e anche rielaborazione del metodo dicotomico, rendeva inutile l'applicazione di schemi euristici ampiamente usati nell' $H A$. Però, come si è già detto, il punto di vista generale del metodo comparativo non è scoperto e per la prima volta 
applicato nel $P A$, invece risulta come frutto del metodo dicotomico usato nell' $H A$. Tuttavia, un'importante caratteristica del $P A$ sta nello sviluppo del metodo comparativo e nell'inquadrarlo in un contesto teoretico più ampio. Come si è detto, secondo Aristotele la physis si divide in varie regioni dei viventi, degli animali. Cioè da una parte viene la possibilità di delimitare analogicamente una regione della realtà (metodo espositivo-dichiarativo), dall'altra in luce di "principi propri" della regione, che esprimono le analogie strutturali delle sostanze, si può passare alla zoologia comparata. Nei libri II-IV del $P A$, dove si svolge il proprio trattato di anatomo-fisiologia comparata sulle parti degli animali, Aristotele cerca le analogie strutturali che definiscono le regioni degli animali e che costituiscono una preziosa chiave per accedere alla comprensione di ogni singola specie (Gotthelf 1987, 167-198).

I principi (archai) della scienza secondo Aristotele hanno una funzione fondamentale in ogni procedimento scientifico. Essi sono strumenti analitici che servono a passare da una conoscenza generale e indeterminata dell'oggetto ad una sua conoscenza specifica e articolata. Essi appartengono ad oggetto in quanto esprimono la modalità del suo specifico essere e dei processi le cose della natura presentano. Da qui la necessità che i principi di ogni scienza siano proprie e specifiche del campo di oggetti indagati. In tal modo i principi non vengono dimostrati, ma piuttosto vengono fornite dall'esperienza (APr. 46a18-9; $A P r$. I. 30; cf. $A P o$. 72a6). La formulazione dei principi primi dall'esperienza avviene in modo particolare grazie al processo induttivo, cioè dalla epagoghé (Phys. 185a9-19) (Marcacci 2009, 215-241). I principi hanno una duplice valenza. Da un lato, essi esprimono le analogie strutturali proprie del campo della realtà, che l'esperienza mostra e l'induzione isola dal contesto dell'indagine. Dall'altro lato, essi servono come strumenti analitici della realtà stessa, cioè in qualche senso come le leggi della natura (Vegetti 1971c, 534).

Anche se Aristotele non sviluppa la discussione sui "principi” della biologia in modo equivalente a quella svolta intorno al problema della causalità, sarebbe troppo semplicistico dire che l'importanza scientifica del $P A$ sta solo nel mettere in luce il problema delle cause. Lo Stagirita non fornisce l'elencazione astratta dei principi dell'anatomia comparata ma li mette nel concreto lavoro della sua elaborazione, perché "occorre trarne un'osservazione generale, che sarà utile sia a proposito della presente questione sia di molte altre che verranno discusse in seguito" (PA 661b26). Si potrebbero descrivere, seguendo l'esposizione di M. Vegetti, almeno nove maggiori principi della teorizzazione scientifica di biologia inclusi nel $P A$ (Vegetti 1971c, 535-540). 
Primo, "la natura adatta infatti gli organi alla funzione, non la funzione agli organi” ( $P A$ 694b13-4). Come si è già detto a proposito della causalità, questo principio non significa un'aprioristica visione del finalismo nell'approccio di Aristotele, ma egli cerca di comprendere, considerando le analogie funzionali fra gli animali, le analogie fra gli organi dei diversi animali. Secondo, "la natura non fa nulla senza scopo" (PA 658a8-9; cf. PA 661b23-4; 691b4; 694a15; 695b19; de Incessu 704b15). Da quanto è stato detto finora, risulta che questo principio non significa che la spiegazione scientifica è determinata dal finalismo assoluto (idea della Natura Divinizzata o presenza del Demiurgo), ma che esiste in ogni caso una stretta relazione causale tra funzione e struttura. Terzo, il principio di compensazione, o degli equivalenti organici o dell'equilibrio organico (presentato in due formulazioni diverse: $P A$ 652a31-3, 665a27-8). Quarto, il principio della divisione del lavoro, esprime il rapporto tra la complessità del modo di vita e organizzazione biologica dei viventi ( $P A$ 683a20-6). Da questo principio consegue la legge secondo cui tanto maggiore la complessità delle funzioni dell'organismo, tanto maggiore la complessità della sua organizzazione. Quinto, la natura "dota di organi atti a servire come strumenti (...) solo o soprattutto gli animali che sono in grado di usarli, e in misura maggiore quelli che possono maggiormente usarli" ( $P A$ 661b26-32; cf. $P A$ 658a23-24). Sesto, "laddove è possibile, un solo principio è infatti meglio che molti. Il cuore è il principio delle vene (...)" (PA 665b14-5). Questo principio è riferito in modo particolare al cuore che costituisce il "principio" psicobiologico dell'organismo. Dal punto di vista metodico, si potrebbe dire che questo principio corrisponde allo sforzo euclideo di ridurre al minimo il numero degli assiomi necessari. Settimo, il principio delle coppie polari ( $P A$ 665a22-5) (Lloyd 1987, 53-63). Ottavo, la continuità della natura, perché la natura passa senza soluzione di continuità delle cose inanimate agli animali (PA 681a12-5; cf. $P A$ 686b29-35). Questo principio esprime una delle più importanti intuizioni aristoteliche, perché si fonda sulla descrizione delle variazioni morfologiche e funzionali che esprimono il passaggio dall'uomo, per il tramite degli animali, fino alle piante (Balme 1987c, 291-312). Di conseguenza questo principio consente ad Aristotele un'elasticità nell'interpretazione degli esseri intermedi fra gli animali e le piante. Il nono principio esprime un certo tipo di antropocentrismo dell'anatomia comparata di Aristotele (PA 656a7-12; 686b2-5; de Incessu 706a19). In realtà Aristotele, sottolineando la posizione eretta dell'uomo e anche la sua partecipazione al divino (intelligenza, nous), cioè la capacità di valorizzare la vita, esprime la peculiarità dell'uomo nel mondo dei viventi ${ }^{4}$. Sembra che i maggiori

4 L'importanza scientifica del tema aristotelico di peculiarità dell'uomo è strettamente collegata con una vivace discussione scientifica sul tema della vita organica nelle sue diverse dimensioni. Sembra che sia possibile recuperare l'uso dell'approccio aristotelico nel contemporaneo dibattito sul problema mente-corpo nel contesto di nuova filosofia meccanicistica della neuroscienza. Tuttavia si deve distinguere 
principi della scienza aristotelica non sono mai imposti in modo aprioristico all'esperienza, ma da essa stessa derivano (Gotthelf 1987, 167-198).

\section{CONCLUSIONE}

Come è stato spiegato fra gli elementi più grandi della ricerca biologica di Aristotele si potrebbe indicare salda unità tra l'anatomia e la fisiologia. Infatti, il discorso aristotelico non è mai né solamente anatomico né solamente fisiologico. Queste due prospettive appaiono compresenti e proprio per questo motivo il suo studio della biologia è così fecondo. Accanto a quest'unità, grazie all'opera di Aristotele, la biologia acquisisce la consapevolezza dell'omologia anatomica e fisiologica tra i diversi animali, ciò trova la sua migliore espressione nella nascita della zoologia comparata come scienza e l'utilizzazione di un ricco materiale empirico. Infatti, le specie citate da Aristotele nell' $H A$ sono circa 450 o 480. Secondo D. Balme $(1987 \mathrm{a}, 16)$ in tutte le opere biologiche sono descritte 560 specie, ma in $H A$ ne sono menzionate 390 . Poi, quanto alle differenze scoperte tra le specie, le due sembrano molto significative: la collocazione dei cetacei fra $\mathrm{i}$ mammiferi e la distinzione tra pesci ossei e pesci cartilaginei, e anche la riproduzione vivipara dei selacei. Riguardo al collocare dei cetacei tra i mammiferi, la genialità di Aristotele si comprende meglio quando si fa riferimento a Linneo, che giunse solo nella decima edizione della sua opera a descriverlo in questo modo. Inoltre, il pensiero biologico di Aristotele tocca il livello scientifico più avanzato nei settori della genetica e dell'embriologia. In questo contesto ci sono due momenti assai significativi: da una parte la distinzione fra lo sviluppo embrionale a partire dall'uovo, la metamorfosi a partire dalla larva (HA I.5), e la splendida descrizione della metamorfosi degli insetti ( $H A$ V.19); e dall'altra parte, l'importante descrizione dello sviluppo dell'uovo e dell'embrione dell'uccello (HA VI.2-3). Si potrebbe anche notare le sue prime intuizioni di psicologia comparata (HA VIII.1). Tuttavia, questo settore del sapere, mediante la teoria delle cause, rivolse il suo sviluppo verso il campo dell'anatomia e della fisiologia, dove le relazioni materia-forma, processo-fine, organo-funzione, trovano le spiegazioni più compatte e più rigorose. Infatti, come si è detto, nel finalismo aristotelico sta la grande forza del suo pensiero. La dottrina aristotelica delle quattro cause non poggia tanto sulla causa finalis da dover escludere le altre, ma la spiegazione aristotelica, che unisce la dinamica della causalità formale con la causalità finale, offre una spiegazione cauta del fenomeno della vita, dove la causa della vita sta nella diversa forma (struttura) assunta dalla materia e non in una diversità di

attentamente quest'approccio dalle diverse forme dell'emergentismo che non sono con esso compatibili (De Haan, 9-38). 
materia. Alla fine, si potrebbe costatare che l'eccezionale capacità di Aristotele a riconoscere una pluralità di piani nei quali si colloca il fenomeno considerato e il costante esercizio di generalizzazione e semplificazione dei problemi studiati, rappresentano la sua grandezza di scienziato e filosofo. La scienza aristotelica presenta una varietà ed elasticità di approcci esplicativi alla realtà (Vegetti 1971b, 121-124; Vegetti 1971c, 514).

Nei secoli successivi la ricerca biologica di Aristotele esercitò un impatto più immediato sul pensiero medico (il progresso della medicina alessandrina del III sec. a.C.) e pertanto poi viene assimilata e subordinata alla scienza medica. La sistematica di Galeno (il cui trattato De usu partium si presenta come perfezionamento del $P A)$ e il lavoro enciclopedico dei medievali ha consolidato questo stato di cose. Grazie alle traduzioni di Guglielmo di Moerbeke e della sintesi $D e$ animalibus di Alberto Magno, nel Duecento, e poi nel Quattrocento grazie al lavoro di Teodoro Gaza, s'inizia la ripresa scientifica rinascimentale della speculazione biologica dello Stagirita. Si dovrebbe sottolineare che il magistero aristotelico nella zoologia comparata rimane saldo fino a tempi di Linneo e Cuvier, perché non muta poi in sostanza nella ricerca biologica il criterio aristotelico adottato della combinazione dei dati anatomici con quelli fisiologici. Tuttavia più vivace e controversa era la riflessione sull'anatomo-fisiologia ed embriologia nei due secoli dell'inizio della scienza moderna (Berti 2004, 197-198).

\section{REFERENCIAS BIBLIOGRÁFICAS}

Aristotele. 2011. Fisica, edited by Roberto Radice. Milano: Biompiani.

- 2013. Metafisica, edited by Giovanni Reale. Milano: Biompiani.

- 2015. L'anima, edited by Giancarlo Movia. Milano: Biompiani.

-. 1924. Aristotle's Metaphysics, edited by William D. Ross. Oxford: Clarendon Press.

—. Gli Analitici Primi, edited by Mario Mignucci. Napoli: Loffredo Editore, 1969.

-. 2007. Analitici secondi, edited by Mario Mignucci. Roma-Bari: Laterza.

Basti, Gianfranco. 2008. Filosofia dell'uomo. Bologna: ESD.

Balme, David M. 1987a. "The place of biology in Aristotle's philosophy". In Philosophical issues in Aristotle's biology, edited by Allan Gotthelf, James G. Lennox, 9-20. Cambridge: CUP.

-. 1987b. "Teleology and necessity". In Philosophical issues in Aristotle's biology, edited by Allan Gotthelf, James G. Lennox, 275-285. Cambridge: CUP. 
-. 1987c. "Aristotle's biology was not essentialist". In Philosophical issues in Aristotle's biology, edited by Allan Gotthelf, James G. Lennox, 291-312. Cambridge, CUP.

Balme. David M. ed. 2002. Historia animalium, volume I, Books I-X: text, edited by Cambridge: CUP.

Barnes, Jonathan, ed. The Complete Works of Aristotle. Princeton-New York, 1991.

Berti, Enrico. 2004. Guida ad Aristotele. Roma-Bari: Laterza.

Bonitz, Hermann. Index Aristotelicus. Graz: Akademische Druck-V. Verlagsanstalt, 1955.

Cherniss, Harold. 1944. Aristotle's Criticism of Plato and the Academy. Baltimore: John Hopkins Press.

Cooper, John M. 1987. "Hypothetical necessity and natural teleology". In Philosophical issues in Aristotle's biology, edited by Allan Gotthelf, James G. Lennox, 243-274. Cambridge: CUP.

De Haan, Daniel. "Hylomorphic Animalism, Emergentism, and the Challenge of the New Mechanist Philosophy of Neuroscience". Scientia et Fides 5/2/ (2017): 9-38.

Gotthelf, Allan. 1987. "First principles in Aristotle's Parts of Animals". In Philosophical issues in Aristotle's biology, edited by Allan Gotthelf, James G. Lennox, 167-198. Cambridge: CUP.

Gotthelf, Allan, ed. 1992. De partibus animalium I and De generatione Animalium I (with passages from II. 1-3). Oxford: Clarendon Press.

Lanza, Diego e Mario Vegetti, eds. Opere biologiche di Aristotele. Torino: UTET, 1971.

Lennox, James G. 2001a. Aristotle's philosophy of biology. Studies in the origins of life science. Cambridge: CUP.

-.2001b. On the parts of animals, translated with a commentary by J.G. Lennox. Oxford: Clarendon Press.

Lloyd, Geoffrey E.R. 1987. "Empirical research in Aristotle's biology". In Philosophical issues in Aristotle's biology, edited by Allan Gotthelf, James G. Lennox, 53-63. Cambridge: CUP.

Marcacci, Flavia. 2009. Alle origini dell'assiomatica: gli Eleati, Aristotele, Euclide. Roma: Aracne.

Pellegrin, Pierre. 1987. "Logical difference and biological difference: the unity of Aristotle's thought". In Philosophical issues in Aristotle's biology, edited by Allan Gotthelf, James G. Lennox, 313-338. Cambridge: CUP.

Rossetti, Livio. 2004. "Empedocle scienziato". In ed. Livio Rossetti, Carlo Santaniello, Studi sul pensiero e sulla lingua di Empedocle, 95-110. Coll. di Studi e Testi 37: Bari. 
Tabaczek, Mariusz. "Emergence and Downward Causation Reconsidered in Terms of the Aristotelian-Thomistic View of Causation and Divine Action". Scientia et Fides 4/1 (2016): 115-134.

Vegetti, Mario. 1971a. "Introduzione”. In Opere biologiche di Aristotele, edited by Diego Lanza, Mario Vegetti, 9-43. Torino: UTET.

-. 1971b. "Origini e metodi della zoologia aristotelica nella Historia Animalium". In Opere biologiche di Aristotele, edited by Diego Lanza, Mario Vegetti, 77128. Torino: UTET.

—. 1971c. "I fondamenti teorici della biologia aristotelica nel De partibus animalium". In Opere biologiche di Aristotele, edited by Diego Lanza, Mario Vegetti, 489-553. Torino: UTET.

Michał Oleksowicz

Facoltà di Teologia Nicolaus Copernicus University ul. Gagarina 37

87-100 Torun (Polonia) https://orcid.org/0000-0001-5591-0579 